\title{
Age-related influences of prior sleep on brain activation during verbal encoding
}

\section{Michelle B. Jonelis ${ }^{1,2}$, Sean P. A. Drummond ${ }^{3,4}{ }^{*}$, Jennifer S. Salamat ${ }^{5}$, Benjamin S. McKenna ${ }^{5}$, Sonia Ancoli-Israel ${ }^{4,5}$ and Mark W. Bondi ${ }^{3,4}$}

' Department of Neurology, Stanford University, Palo Alto, CA, USA

2 Howard Hughes Medical Institute, Chevy Chase, MD, USA

${ }^{3}$ Psychology Services, VA San Diego Healthcare System, San Diego, CA, USA

${ }^{4}$ Department of Psychiatry, University of California San Diego, San Diego, CA, USA

${ }^{5}$ Research, VA San Diego Healthcare System, San Diego, CA, USA

\section{Edited by:}

Linda J. Larson-Prior, Washington

University School of Medicine in St. Louis, USA

\section{Reviewed by:}

Ramesh Vijay, University of Chicago Medical Center, USA

Lisa Marshall, University of Luebeck, Germany

\section{*Correspondence}

Sean P. A. Drummond, Psychology Services, VA San Diego Healthcare System, 3350 La Jolla Village Drive MC151B, San Diego, CA 92161, USA. e-mail:drummond@ucsd.edu
Disrupted sleep is more common in older adults (OLD) than younger adults (YOUNG), often co-morbid with other conditions. How these sleep disturbances affect cognitive performance is an area of active study. We examined whether brain activation during verbal encoding correlates with sleep quantity and quality the night before testing in a group of healthy OLD and YOUNG. Twenty-seven OLD (ages 59-82) and 27 YOUNG (ages 19-36) underwent one night of standard polysomnography. Twelve hours post-awakening, subjects performed a verbal encoding task while undergoing functional magnetic resonance imaging. Analyses examined the group (OLD vs. YOUNG) by prior sleep quantity (total sleep time, TST) or quality (sleep efficiency, SE) interaction on cerebral activation, controlling for performance. LongerTST promoted higher levels of activation in the bilateral anterior parahippocampal in OLD and lower activation levels in the left anterior parahippocampus in YOUNG. Greater SE promoted higher activation levels in the left posterior parahippocampus and right inferior frontal gyrus in YOUNG, but not in OLD. The roles of these brain regions in verbal encoding suggest, in OLD, longer sleep duration may be linked to the ability to engage in functional compensation during cognitive challenges. By contrast, in YOUNG, shorter sleep duration may necessitate functional compensation to maintain cognitive performance, similar to what is seen following acute sleep deprivation. Additionally, in YOUNG, better sleep quality may improve semantic retrieval processes, thereby aiding encoding.

Keywords: aging, sleep, fMRI, cerebral activation, cognitive performance, verbal encoding

\section{INTRODUCTION}

Disrupted sleep is more common in older adults (OLD) than younger adults (YOUNG), often co-morbid with other conditions. Relative to YOUNG, the sleep of OLD is generally characterized by decreased total sleep time (TST), poorer sleep efficiency (SE), and a smaller percentage of time spent in slow wave sleep (Ohayon et al., 2004). Similarly, relative to YOUNG, OLD on average tend to perform worse on many cognitive tasks. Learning and memory are among the most widely studied cognitive domains showing age-related declines (Johns, 1991; Salthouse and Ferrer-Caja, 2003; Cabeza et al., 2004; Kemps and Newson, 2006). Although the general trend is toward a decline with age, OLD show greater variability than YOUNG in both sleep and cognition, with some OLD showing little or no decline relative to YOUNG (Middleton and Strick, 2002; Ohayon et al., 2004). While both sleep and cognitive performance measures tend to decrease with age, if and how sleep changes in aging might affect cognitive function is not well known. Since experimental sleep deprivation studies show sleep loss is associated with performance decline in most cognitive domains in YOUNG, there has been speculation about whether the decline in cognitive performance with age could be explained by the corresponding decline in sleep with age.

Multiple studies examining the relationship between sleep and cognition in generally healthy, non-demented OLD have concluded sleep does influence some aspect of cognition. For example, two studies using actigraphy in a cohort of over 3000 older women found clinically significant sleep fragmentation, indexed by a reduced percentage of time during an overnight sleep period actually spent asleep, were associated with lower scores on mental status and executive function tests (Blackwell et al., 2006; Nielsen and Levin, 2007; Yaffe et al., 2007). Other studies reported increases in obstructive sleep apnea severity over time, associated with increased sleep fragmentation, were also associated with a decline in mental status exam scores (Cohen-Zion et al., 2001, 2004). Specific to learning and memory, a large scale questionnairebased study reported subjectively impaired sleep was associated with decreased immediate and delayed verbal recall (Ansiau et al., 2008). Additionally, three nights of experimental sleep restriction in OLD reduced verbal recall performance (Stenuit and Kerkhofs, 2008). Together, these studies suggest the quality or quantity of sleep in OLD is associated with cognitive performance. 
However, the bulk of evidence regarding sleep-cognition associations is based largely on self-report and questionnaire methods that are prone to biases and a variety of confounds or through experimentally imposed sleep deprivation.

A strategy to examine the sleep-cognition relationship more directly than those methods outlined above would be to examine neurophysiological correlates of cognition (e.g., neuroimaging or EEG) during administration of standardized tests. For example, functional magnetic resonance imaging (fMRI) studies comparing cerebral responses in OLD vs. YOUNG have often reported OLD show more bilateral and/or increased activation during cognitive performance (Cabeza et al., 1997; Reuter-Lorenz et al., 1999; Cabeza, 2002; Reuter-Lorenz, 2002; Han et al., 2009). This increased bilateral activation has been shown by several authors to correlate with better cognitive performance and has therefore been referred to as "compensatory recruitment" (Cabeza et al., 2002; Reuter-Lorenz, 2002; among other). This pattern is strikingly similar to that seen in YOUNG following experimental sleep deprivation, when compared to YOUNG well-rested (Drummond et al., 2000, 2005; Drummond and Brown, 2001; Stricker et al., 2006). The parallels between increased activation observed in OLD relative to YOUNG and increased activation seen in sleep deprived YOUNG relative to well-rested YOUNG suggest similar compensatory mechanisms may occur in both aging and sleep deprivation. This, in turn, further supports the suggestion from the studies outlined above that some of the cognitive changes seen in OLD may relate to changes in sleep. However, no study thus far has looked at how normal sleep changes with aging affect brain activation during cognitive tasks.

Here, we examine whether fMRI-derived measures of brain activation during a verbal learning (VL) task correlate with sleep quantity (TST) and/or quality (SE) the night before scanning in a group of healthy OLD and YOUNG. Our aim was to identify if activation in any brain regions are related to prior sleep quantity and/or quality, and if these relationship differ in OLD and YOUNG. Our hypothesis was that lower quantity and quality of sleep would result in greater recruitment of brain regions involved in VL for OLD, but not YOUNG.

\section{MATERIALS AND METHODS SUBJECTS AND SCREENING}

The study was approved by the UCSD Human Research Protection Program and the VA San Diego Healthcare System R\&D Committee, and all subjects provided written informed consent. A total of 62 adults (29 YOUNG, 33 OLD) participated in the study. One YOUNG and six OLD were excluded due to excessive movement during scanning. An additional YOUNG was excluded for extremely poor sleep the night prior to testing. Thus, data for 54 subjects ( 27 OLD and 27 YOUNG) are reported here. There were no significant differences between baseline characteristics of the older and younger subjects except on age (Table 1). Subjects were medically healthy and free of current and past psychiatric diagnoses as determined by interview, history, and physical examination, Structured Clinical Interview for DSM-IV (SCID), routine laboratory work, and urine toxicology screens. We only enrolled subjects with a BMI $<35$ and without any self- or collateral-report of sleep apnea symptoms (e.g., frequent loud snoring, witnessed
Table 1 | Subject demographics.

\begin{tabular}{llll}
\hline & Group & Mean & Range \\
\hline Age (years) & Older & $67.7 \pm 6.0$ & $59-82$ \\
& Younger & $27.4 \pm 4.3$ & $19-36$ \\
Gender & Older & $21 \mathrm{~F}$ & \\
& Younger & $15 \mathrm{~F}$ & \\
Ethnicity/race & Older & 25 Non-Hispanic & 26 White \\
& Younger & 22 Non-Hispanic & 21 White \\
Education (years) & Older & $15.4 \pm 2.5$ & $10-20$ \\
\multirow{2}{*}{ BMI } & Younger & $15.0 \pm 2.3$ & $12-20$ \\
& Older & $25.1 \pm 3.5$ & $19-33$ \\
& Younger & $25.4 \pm 4.1$ & $20-33$ \\
\hline
\end{tabular}

Sample size $=27$ subjects in each group.

Table 2 | Neuropsychological test scores.

\begin{tabular}{lll}
\hline Test & Older & Younger \\
\hline Dementia rating scale & $56.4 \pm 3.0$ & Not given \\
CVLT 1-5 T-score & $59.7 \pm 8.9$ & $59.9 \pm 11.7$ \\
CVLT long delay recognition accuracy & $99.8 \pm 1.2$ & $100.0 \pm 0$ \\
Logical memory 1 scaled recall & $14.0 \pm 2.4$ & $13.2 \pm 2.8$ \\
Logical memory 2 scaled recall & $14.0 \pm 2.3$ & $14.0 \pm 2.7$ \\
\hline
\end{tabular}

There were no group differences on age-matched norms for any test.

apnea, etc.). We also excluded any subject with an apnea-hypopnea index of PLM with arousal index $>10$ on a screening sleep study. Stable doses of commonly prescribed medications, including antihypertensives, hormone replacement therapy, and oral contraceptive pills, were permitted during the study. Anyone reporting $>14$ alcoholic drinks/week was excluded (OLD reported a mean of $2.9 \pm 3.5$ drinks/week; YOUNG reported a mean of $2.5 \pm 3.5$ drinks/week). Eligible subjects reported habitually sleeping 7-9 h per night with no more than one nap per week. For 7-10 days prior to the study, subjects completed daily sleep diaries and maintained an agreed-upon sleep-wake schedule matching their habitual sleep schedule and specifically excluded naps.

Subjects were administered standard neuropsychological tests to insure their verbal memory function was in the normal range and the groups were matched for performance. Subjects were required to score within normal limits for age on all measures for inclusion in the study (Table 2), and the two groups did perform equivalently on each of these age-normed neuropsychological tests. In particular, we assessed immediate recall and delayed recognition for a list of words with the California Verbal Learning Test-II (Delis et al., 2000) and the ability to encode and recall a story with the Logical Memory subset of the Weschler Memory Scale-III (Wechsler, 1997). For the OLD group only, we also administered the Dementia Rating Scale and required subjects to score in the normal range (Mattis, 1988).

\section{EXPERIMENTAL PERIODS}

Following the week of habitual sleep monitoring at home, subjects underwent two consecutive nights of polysomnography, the 
first of which served as a screen for unreported sleep disorders. The timing of sleep on both nights was matched to the habitual schedule previously maintained at home. Polysomnography was collected on Grass Heritage EEG systems and digitally recorded with a sampling rate of $256 \mathrm{~Hz}$, a low frequency filter of $0.3 \mathrm{~Hz}$, and a high frequency filter of $100 \mathrm{~Hz}$. For scoring of sleep stages, $\mathrm{C} 3$ and $\mathrm{O} 1$ served as the primary electrode sites, with contralateral $\mathrm{C} 4$ and $\mathrm{O} 2$ serving as back-up sites in case of artifacts in the primary sites. Twelve hours after waking from the second night, subjects completed a VL task while undergoing fMRI. All subjects were previously trained on the VL task prior to the two nights sleeping in the lab.

\section{EXPERIMENTAL TASK}

During each fMRI session, stimuli were presented visually via an LCD projector onto a screen placed at the foot of the MRI bed that subjects viewed through a mirror fitted to the head coil. The alternating block design task consisted of two visually identical parts. During the entire task, subjects saw stimuli presented one at a time, each for $4 \mathrm{~s}$ followed by $1 \mathrm{~s}$ of a fixation asterisk. During memorization blocks, subjects saw nouns and were instructed to actively memorize the words for later testing. To aid encoding, subjects pressed a button on a hand-held button box (Current Designs, Philadelphia) to indicate whether the word referred to a concrete item or an abstract concept. For baseline blocks, subjects saw strings of a single, repeated letter and were instructed to press a button to indicate the case of the letters (CAPS vs. lowercase). The VL task contained six memorization blocks and seven baseline blocks (the task started and ended with baseline). Each block started with directional prompts for $2.5 \mathrm{~s}$ and lasted a total of $22.5 \mathrm{~s}$. The entire task lasted $300 \mathrm{~s}$ (including $6 \mathrm{~s}$ at the start for fMRI data not analyzed due to partial saturation effects). A total of 10 different versions of the VL task were developed, with each list matched for recallability, word length, concreteness, and imagery. Previous pilot studies show that the versions provide similar recall rates and subjects reached stable performance after one practice session (Drummond et al., 2005; Stricker et al., 2006).

At the conclusion of the task, while still in the fMRI scanner, subjects were asked to freely recall as many words as possible. Subjects were then administered a series of questions: the Karolinska Sleepiness Scale (a 9-point anchored scale assessing state sleepiness ranging, from "1 Extremely alert" to "9 Extremely sleepy - fighting sleep"; Akerstedt and Gillberg, 1990), and 10-point Likert scale questions assessing the following subjective factors: task difficulty, ability to concentrate, effort put into the task, and motivation to perform the task well. These Likert scales were developed in-house and are discussed in more detail elsewhere (Drummond et al., 2005; Ayalon et al., 2006). After completion of the entire scanning session, subjects were given a written recognition memory test.

Data were acquired with a General Electric 3T scanner. Functional images consisted of 120 gradient echo EPI images (TR: $2.5 \mathrm{~s}, 3.91 \mathrm{~mm}^{2}$ in-plane resolution) of thirty-two $4 \mathrm{~mm}$ axial slices covering the whole brain and measuring the blood oxygen level-dependent (BOLD) signal. Functional data were aligned with high-resolution anatomical images (FSPGR: $1 \mathrm{~mm}^{3}$ resolution).

\section{DATA ANALYSIS}

Overnight sleep data were scored by experienced, certified PSG technicians according to standard criteria. TST and SE from the night immediately prior to testing served as the sleep variables of interest. TST is defined as the total minutes of sleep obtained between the time when subjects first attempted to fall asleep (lights out) and when the sleep study ended in the morning (lights on). As mentioned above, lights out and lights on were set to match each subject's habitual schedule as closely as possible. The time between lights out and lights on comprises Time in Bed. SE is a measure of how consolidated an individual's sleep is, and is defined as TST/Time in Bed. Thus, it represents the proportion of time one is in bed trying to sleep that one is actually asleep.

Behavioral data were analyzed by regressing group (OLD vs. YOUNG), prior sleep (i.e., TST or SE), and the group-by-prior sleep interaction on performance. Behavioral outcome variables of interest were the number of words immediately recalled after the task (free recall) and discriminability index $\left(\mathrm{D}^{\prime}\right)$ for recognition memory ( $\mathrm{D}^{\prime}$ takes into account the number of words correctly identified and the number of foils chosen). Post-scan questionnaire data were analyzed with independent samples $t$-tests.

Functional magnetic resonance imaging data were processed and analyzed with analysis of functional neuroimages (AFNI; Cox, 1996) in a two-step procedure: individual time-course analysis followed by group statistical analysis. After motion coregistration, individual time-course BOLD signal data were fit to a design matrix using the general linear model (GLM). Parameters estimated from the design matrix represented the constant, linear drift, six motion-correction parameters derived from the motion coregistration step (three relational and three translational movement directions), and the reference function. The reference function of interest in the GLM was a representation of the task design (memorization vs. baseline) convolved with an idealized hemodynamic response function (Cohen, 1997). The parameter used in group analyses was the regression coefficient associated with this reference function. Datasets were smoothed with a Gaussian filter of 4.0 -mm full-width-half-maximum and transformed to standard atlas coordinates (Talairach and Tournoux, 1988).

Group analyses used a search region of interest (ROI) protocol (Eyler Zorrilla et al., 2003; Drummond et al., 2005). Our search region consisted of the following areas bilaterally: inferior frontal gyrus (IFG), inferior and superior parietal lobes, and hippocam$\mathrm{pal} /$ parahippocampal gyri. The coordinates of the Talairach and Tournoux atlas within AFNI defined these regions (Talairach and Tournoux, 1988). We chose to inspect these regions based on our previous findings of compensatory recruitment during this task following TSD (Drummond et al., 2000) as well as from studies in healthy aging (Cabeza, 2002; Cabeza et al., 2002; Reuter-Lorenz, 2002). We used a cluster threshold method to protect against Type I error (Forman et al., 1995), reporting only clusters containing $\geq 6$ contiguous voxels $\left(384 \mathrm{~mm}^{3}\right)$, each activated at $p \leq 0.05$. This procedure maintained a whole brain alpha level $=0.05$.

The group analyses of the neuroimaging data regress group (OLD vs. YOUNG), prior sleep (i.e., TST or SE), recognition memory performance, and the group-by-prior sleep interaction onto BOLD units. Recognition memory was included because of its association with BOLD signal (Drummond et al., 2005) and 
because we sought to examine whether prior sleep contributed to cerebral activation beyond that related to recognition memory. This decision also helped account for the fact behavioral performance was not equivalent between the two groups (see Results). The main parameter of interest from the group analysis was the interaction effect, with the other effects statistically partialed out. Follow-up analyses consisted of analyzing the effect of sleep (i.e., TST or SE) on cerebral responses within each group to better understand the source of significant interactions.

\section{RESULTS}

\section{POLYSOMNOGRAPHY}

Consistent with the literature, our healthy OLD showed significantly shorter TST and lower SE compared to YOUNG, as measured by PSG the night before testing (Table 3 ).

\section{BEHAVIORAL DATA}

Table 4 shows behavioral performance and subjective measures from the fMRI sessions. OLD performed worse than YOUNG on both recall and recognition memory. There were no significant group-by-prior sleep interaction effects on free recall or recognition memory $(p>0.05)$. The groups did not differ on any subjective measure related to sleepiness or task performance $(p>0.05)$.

\section{FMRI DATA}

The group-by-prior sleep interaction, independent of performance, had a significant effect on BOLD activation in four brain regions. Table 5 provides the anatomical location, Brodmann's areas (BA), volume, and maximum effect size (partial $r^{2}$ ) information for the clusters showing an interaction. Table 6 provides the parameters estimates, $t$-value, and significance for each parameter in the models associated with each significant cluster.

For TST, the group-by-prior sleep interaction was significant in the bilateral anterior parahippocampal gyri (APH; Figure 1). The interaction was explained by a strong, positive correlation between TST and cerebral responses in OLD, with no significant correlation in the YOUNG in the right $\mathrm{APH}$, and a negative correlation in the YOUNG in the left APH.

For SE, the group-by-prior sleep interaction was significant in the left posterior parahippocampal gyrus $(\mathrm{PPH})$ and right (IFG;
Figure 2). Here, the interaction was explained by a strong, positive correlation between SE and cerebral responses in the YOUNG, with no significant correlation in the OLD.

\section{DISCUSSION}

We hypothesized lower quantity and quality of sleep would result in greater cerebral responses (index by increased BOLD signal) in brain regions involved in verbal encoding for OLD but not for YOUNG (i.e., there would be brain regions showing a group-by-prior sleep interaction that would be explained by a negative correlation in OLD with no correlation in the YOUNG). We

Table 4 | Behavioral performance and subjective measures during fMRI.

\begin{tabular}{llll}
\hline & Measure (scale) & $\begin{array}{l}\text { Older } \\
\text { adults }\end{array}$ & $\begin{array}{l}\text { Younger } \\
\text { adults }\end{array}$ \\
\hline \multirow{2}{*}{ VL performance } & \#Words recalled* & $4.4 \pm 3.2$ & $8.9 \pm 3.9$ \\
\cline { 2 - 3 } Questionnaires & Recognition memory D' * & $2.4 \pm 1.0$ & $3.6 \pm 1.1$ \\
& Karolinska sleepiness scale (1-9) & $3.6 \pm 1.7$ & $3.6 \pm 1.6$ \\
& Concentration (1-10) & $7.6 \pm 2.2$ & $8.5 \pm 1.3$ \\
& Task difficulty (1-10) & $7.1 \pm 2.6$ & $6.3 \pm 2.8$ \\
& Motivation (1-10) & $9.2 \pm 1.6$ & $9.3 \pm 1.1$ \\
& Effort (1-10) & $9.2 \pm 1.1$ & $9.2 \pm 1.1$ \\
\hline
\end{tabular}

*Significant difference between groups, $p<0.001$.

Table 5 | Brain regions showing a significant group $\times$ prior sleep interaction.

\begin{tabular}{llllll}
\hline & $\begin{array}{l}\text { Anatomical } \\
\text { location }\end{array}$ & BA & $\begin{array}{l}\text { Volume } \\
\left(\mathbf{m m}^{\mathbf{3}}\right)\end{array}$ & Center $(\boldsymbol{x}, \mathbf{y}, \mathbf{z})$ & $\begin{array}{l}\text { Maximum } \\
\text { effect } \\
\text { size }\left(\boldsymbol{r}^{\mathbf{2}}\right)\end{array}$ \\
\hline TST & R APH & R 35 & 1088 & $26,-14,-18$ & 0.32 \\
& L APH & L 36 & 896 & $-32,-22,-19$ & 0.31 \\
SE & L PPH & L 36 & 1344 & $-26,-41,-6$ & 0.29 \\
& R IFG & R 45/46 & 448 & $42,26,9$ & 0.16 \\
\hline
\end{tabular}

$A P H$, anterior parahippocampus; $P P H$, posterior hippocampus; IFG, inferior frontal gyrus; $R$, right; $L$, left.

Table 3 | PSG-measured sleep from night prior to fMRI tests.

\begin{tabular}{|c|c|c|c|c|}
\hline \multirow[t]{2}{*}{ Measure } & \multicolumn{2}{|c|}{ Older adults $(n=27)$} & \multicolumn{2}{|c|}{ Younger adults ( $n=27$ ) } \\
\hline & Mean \pm SD & Range & Mean \pm SD & Range \\
\hline Total sleep time ${ }^{* *}$ & $391.8 \pm 51.6 \mathrm{~min}$ & $280-479 \min$ & $437.3 \pm 35.4 \mathrm{~min}$ & $361-512 \mathrm{~min}$ \\
\hline Sleep efficiency** & $85.0 \pm 8.6 \%$ & $67-96 \%$ & $92.2 \pm 4.4 \%$ & $82-98 \%$ \\
\hline Sleep latency & $14.6 \pm 18.6 \min$ & $1-67 \mathrm{~min}$ & $11.1 \pm 11 \mathrm{~min}$ & $1-43.5 \mathrm{~min}$ \\
\hline Wake after sleep onset ${ }^{*}$ & $49.7 \pm 29.2 \mathrm{~min}$ & $11-116 \mathrm{~min}$ & $23.2 \pm 18.3 \mathrm{~min}$ & $5-75 \mathrm{~min}$ \\
\hline Stage $1 \%$ & $4.9 \pm 2.3 \%$ & $1.4-11.4 \%$ & $4.6 \pm 2.4 \%$ & $1.6-12.4 \%$ \\
\hline Stage $2 \% * *$ & $65.8 \pm 8.5 \%$ & $44.4-78.4 \%$ & $53.2 \pm 7.2 \%$ & $34.0-68.0 \%$ \\
\hline Slow wave sleep (stage $3+4) \% * *$ & $8.8 \pm 9.1 \%$ & $0-29.0 \%$ & $17.7 \pm 7.7 \%$ & $1.0-35.0 \%$ \\
\hline REM\%* & $20.5 \pm 6.5 \%$ & $3.4-33.2 \%$ & $24.6 \pm 4.8 \%$ & $15.1-32.1 \%$ \\
\hline
\end{tabular}

${ }^{*} p<0.05 ;{ }^{*} p<0.001$ 
Table 6 | Statistical results of fMRI regression models.

\begin{tabular}{llrrc}
\hline Brain region & Independent variables & Beta & \multicolumn{1}{l}{ T } & Sig. \\
\hline R APH & Recognition D' & 0.38 & 2.80 & 0.007 \\
& Group & 0.24 & 1.66 & 0.104 \\
& Total sleep time & -0.43 & -1.84 & 0.073 \\
& Interaction (group $\times$ TST) & 0.83 & 3.81 & $<0.001^{* *}$ \\
LAPH & Recognition D' & 0.22 & 1.59 & 0.119 \\
& Group & -0.16 & -1.09 & 0.279 \\
& Total sleep time & -0.55 & -2.34 & 0.023 \\
& Interaction (group $\times$ TST) & 0.87 & 4.03 & $<0.001^{* *}$ \\
LPPH & Recognition D' & 0.13 & 0.85 & 0.4 \\
& Group & 0.43 & 2.68 & 0.01 \\
& Sleep efficiency & 1.06 & 3.31 & 0.002 \\
R IFG & Interaction (group $\times$ SE) & -0.98 & -3.33 & $0.002^{*}$ \\
& Recognition D' & -0.08 & -0.54 & 0.593 \\
& Group & -0.07 & -0.42 & 0.676 \\
& Sleep efficiency & 0.93 & 2.81 & 0.007 \\
& Interaction (group $\times$ SE) & -0.87 & -2.86 & $0.006^{*}$ \\
& & &
\end{tabular}

$A P H$, anterior parahippocampus; $P P H$, posterior hippocampus; IFG, inferior frontal gyrus; $R$, right; $L$, left.

${ }^{* *} p<0.01 ;{ }^{* * *} p<0.001$.

$f M R I$ regression analyses found four regions (see Figures 1 and 2; Table 5) where the interaction of group and prior sleep predicted BOLD activation, after partialing out group (OLD vs. YOUNG), prior sleep (i.e., TST or SE), and performance.

found there were indeed differences in the influence of prior sleep on cerebral responses to verbal encoding for OLD vs. YOUNG and these were above and beyond the correlation between performance and cerebral responses. However, the interactions were not explained in the manner hypothesized. Rather, longer TST were correlated with higher activation levels in bilateral APH in OLD but lower activation levels in YOUNG. On the other hand, greater SE was correlated with higher activation levels in the left $\mathrm{PPH}$ and the right IFG in YOUNG (but not OLD). Together, these data suggest greater sleep may be associated with acute compensatory recruitment responses in OLD, while more consolidated sleep is associated with such responses in YOUNG. To our knowledge, this is the first fMRI study to look at the effects of prior, habitual levels of sleep on brain activation in OLD and YOUNG.

\section{PRESUMED FUNCTIONS OF INVOLVED BRAIN REGIONS}

The bilateral APH are known to be essential for memory encoding, with the left side typically more involved in verbal encoding (Rosazza et al., 2009). Previous fMRI studies showed OLD often display more bilaterality in cerebral responses than YOUNG (Cabeza et al., 2002; Reuter-Lorenz, 2002; van der Veen et al., 2006; Han et al., 2009). Consistencies in the pattern of increased bilateral fMRI activity across multiple studies and with a variety of behavioral tasks led Cabeza (2002) to propose a model of hemispheric asymmetry reduction in older adults (HAROLD). The HAROLD model argues the observed hemispheric asymmetry normally observed in YOUNG becomes more bilateral in highperforming OLD and thus reflects functional compensation in the face of age-related declines in neural efficiency (see also Han et al., 2009; for discussion). If we assume higher levels of bilateral APH
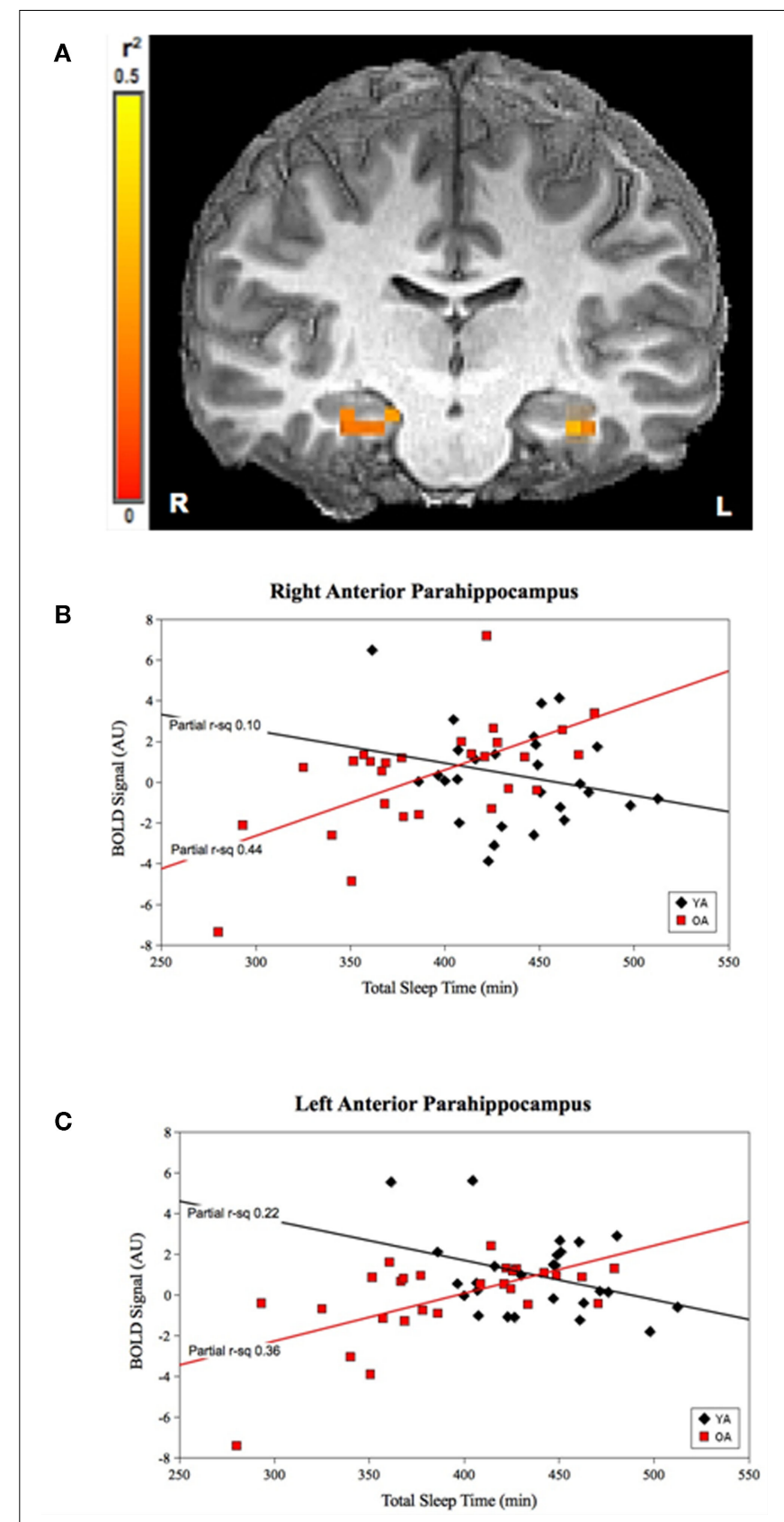

FIGURE 1 | Group-by-prior sleep interaction for TST. Bilateral anterior parahippocampal regions showing a group-by-TST interaction. (A) Color scale represents $r^{2}$ associated with the interaction term in the statistical model (max $\left.r^{2}=0.5\right)$. (B,C) Graphs show a strong, positive TST-BOLD correlation in OLD (both regions $p<0.001$ ), with no significant correlation for the YOUNG $(p>0.05)$ in the right APH and a significant negative correlation for the YOUNG in the left APH $(p<0.05)$. (Effect sizes are partial $r^{2}$ for OLD and YOUNG separately, accounting for effects of performance and the main effects of group and TST.)

activation in our OLD represents functional compensation, our results suggest that perhaps, in OLD, sufficient prior sleep quantity may be a necessary pre-condition for successful functional compensation. 

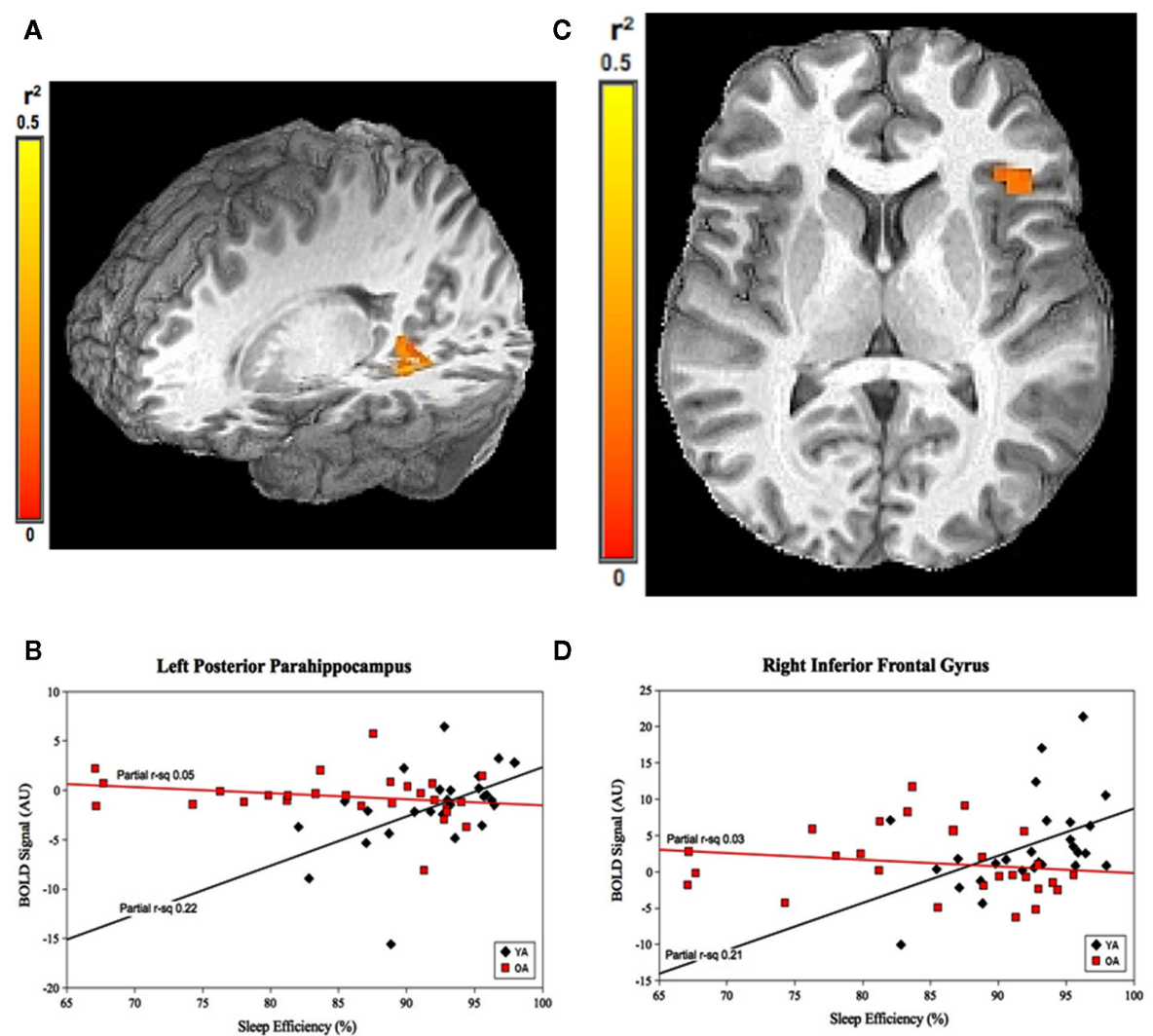

FIGURE 2 | Group-by-prior sleep interaction for SE. Left posterior parahippocampal gyrus and right inferior frontal gyrus regions showing group-by-SE interaction. (A,C) Color scale represents $r^{2}$ associated with the interaction term in the statistical model $\left(\max r^{2}=0.5\right)$. (B,D) Graphs
Younger adults have been shown to engage functional compensation during verbal encoding tasks after sleep deprivation via increased or newly bilateral activation, similar to what is seen when comparing normally rested OLD to normally rested YOUNG (Drummond et al., 2000, 2005; Drummond and Brown, 2001; Stricker et al., 2006). Since left APH activation is strongly associated with verbal encoding in YOUNG (Rosazza et al., 2009), the negative correlation of TST with left APH activation may actually suggest lower TST necessitates mild functional compensation during VL in YOUNG.

The role of the left $\mathrm{PPH}$ region (showing higher activation levels in the YOUNG, but not OLD, with greater SE) in VL and memory is less well characterized than the anterior region. Work by Qin et al. (2009) suggests the PPH is involved in memory retrieval more than encoding. Prince et al. (2005) found left PPH activity was associated with retrieval success. Because we only examined the encoding phase of our task, we hypothesize left PPH activity in our YOUNG may be involved in retrieving information related to the words presented. This hypothesis is supported by Mestres-Misse et al. (2008) who found the PPH to be involved in semantic associative processing. Perhaps in our YOUNG, more consolidated sleep allowed subjects to better retrieve semantic information related to the words they were learning. Such semantic elaboration, in show a strong, positive SE-BOLD correlation for the YOUNG (both $p<0.05$ ), with no significant correlation for the OLD (both $p>0.05$ ). (Effect sizes are partial $r^{2}$ for OLD and YOUNG separately, accounting for the effects of performance.) turn, may have aided the abstract/concrete judgment required, buttressed encoding strategies, or both.

Right IFG also showed higher activation levels in YOUNG, but not OLD, with greater SE. This area may be playing a similar role to PPH in YOUNG by being involved in semantic categorization. Prior studies have found areas near the right IFG to be related to: (a) judgments about metaphoric but not literal semantic relatedness of words (Stringaris et al., 2006); (b) meaning-based processing tasks (Fletcher et al., 2002); and (c) item familiarity during a memory encoding task (Mitchell et al., 2008). Furthermore, there are functional connections between $\mathrm{PPH}$ and areas near right IFG region (Kahn et al., 2008), suggesting a possible relationship between the increased PPH and IFG activation in our YOUNG. Lichter and Cummings (2001) have also found indirect connections between IFG and the hippocampus via the basal ganglia and thalamus. Together, the above findings suggest, in our YOUNG, better SE may have allowed for improved semantic retrieval resulting in higher activation levels in both left $\mathrm{PPH}$ and right IFG.

\section{DIFFERENCES BETWEEN OLD AND YOUNG}

A novel finding from our study is cerebral responses to the verbal encoding task were affected differently by prior sleep in OLD 
and YOUNG. This result suggests different aspects of prior sleep acutely impact the brain's response to cognitive challenges as we age. Here, OLD seemed less sensitive to the effects of poor SE than YOUNG. One possible explanation for this difference is mildly decreased SE is a common and not necessarily pathological aspect of normal aging. Indeed, the seminal meta-analysis of age-related sleep changes found reduced SE was the most common sleep alteration in adults over 60-years-old. Cognitive performance of YOUNG, on the other hand, has been shown in other studies to be sensitive to fragmented sleep. For example, Howell et al. (2004) and Pilcher and Walters (1997) both found relationships between academic performance in high school or college and sleep quality. Another age-related difference is that, while prior sleep produced larger effects sizes in OLD than YOUNG, the effects of prior sleep seem be more multi-faceted in YOUNG. Better SE seems to have aided semantic processing in YOUNG, and longer TST promoted neural efficiency within left APH (to the extent that lower activation levels, while controlling for performance, can be interpreted as neural efficiency). Thus, in YOUNG, better sleep seems to both promote efficiency of the main cognitive process tested here (i.e., encoding) and facilitate ancillary cognitive processes (i.e., semantic retrieval) that likely aided performance.

The age-related differences in the effects of prior sleep reported here have direct implications for potential interventions aimed at maximizing cognitive function. For example, in OLD, clinicians involved in cognitive rehabilitation or efforts to improve memory performance may wish to encourage increased sleep time in their patients as a way to promote more robust cerebral responses to encoding challenges. Those working with YOUNG, for example to improve academic performance, may instead wish to focus on interventions designed to maximize the consolidation of sleep.

\section{LIMITATIONS}

A few limitations of this study should be acknowledged. First, our OLD were ostensibly quite healthy. While this aids internal validity by reducing the probability that medical co-morbidities are responsible for the findings, it may impact generalizability to some extent. Similarly, both groups averaged a college level education and none of the subjects in either group had frankly pathological sleep. Future studies may wish to include a wider range of subjects, in terms of both cognitive ability and sleep, to further explore the relationship between sleep and cerebral responses to cognitive challenges. Second, we only examined the influence of sleep on a single night preceding testing. Bed time and wake time

\section{REFERENCES}

Akerstedt, T., and Gillberg, M. (1990). Subjective and objective sleepiness in the active individual. Int. J. Neurosci. 52, 29-37.

Ansiau, D., Wild, P., Niezborala, M., Rouch, I., and Marquie, J. C. (2008). Effects of working conditions and sleep of the previous day on cognitive performance. Appl. Ergon. 39, 99-106.

the night before testing were based on subjects' habitual, at home sleep schedules, but we nonetheless cannot glean with certainty that our single night sleep data fully reflects the longer-term sleep patterns of these subjects. Future studies may wish to examine sleep over more nights to determine the impact of longer-term sleep quantity/quality on cerebral responses.

\section{CONCLUSION}

In summary, our study used a verbal encoding task to examine whether the quantity and quality of sleep during the night immediately preceding testing affected the cerebral response to this cognitive challenge differently for OLD and YOUNG. We found four distinct brain regions that were differentially sensitive to prior sleep, independent of the association between cerebral responses and performance. Longer TST promoted higher BOLD activation in the bilateral APH in OLD and lower BOLD activation in the left APH in YOUNG. Greater SE promoted higher BOLD activation levels in the left PPH and the right IFG in YOUNG but not in OLD. The roles of these brain regions in verbal encoding suggest different mechanisms by which sleep may influence brain function in older vs. younger adults. In OLD, greater sleep quantity appears linked to greater engagement of functional compensation during cognitive challenges. By contrast, in YOUNG, lower sleep quantity may necessitate functional compensation to maintain cognitive performance, similar to what is seen with acute sleep deprivation. Additionally, in YOUNG, better sleep quality may improve semantic retrieval processes, thereby aiding encoding. OLD with longer TST may have improved cognitive function over their peers with shorter TST, whereas objectively lower SE in the absence of sleep complaints or other sleep pathology may not play as great a role in cognition in this group as previously thought. This suggests that future studies of brain function in OLD may benefit from accounting for variations in TST and/or excluding those subjects with abnormally low TST the night prior to testing.

\section{ACKNOWLEDGMENTS}

This work was supported by National Institute on Aging (RO1 AG024506, AG08415, R01 AG012674, and K24 AG02643); National Institutes of Health (M01 RR00827); Alzheimer's Association (IIRG 07-59343); Howard Hughes Medical Institute (Michelle B. Jonelis was an HHMI Medical Research Training Fellow); and the Veterans Affairs San Diego Center of Excellence for Stress and Mental Health. We would like to acknowledge Robert L. Wang, Lesley A. Wetherell, and Kathy Resovsky, RN, for their invaluable contributions to this study.

function in older women: the study of osteoporotic fractures. J. Gerontol. A Biol. Sci. Med. Sci. 61, 405-410.

Cabeza, R. (2002). Hemispheric asymmetry reduction in older adults: the HAROLD model. Psychol. Aging 17, 85-100.

Cabeza, R., Anderson, N. D., Locantore, J. K., and McIntosh, A. R. (2002). Aging gracefully: compensatory brain activity in high-performing older adults. Neuroimage 17, 1394-1402.

Cabeza, R., Daselaar, S. M., Dolcos, F., Prince, S. E., Budde, M., and Nyberg, L. (2004). Task-independent and task-specific age effects on brain activity during working memory, visual attention and episodic retrieval. Cereb. Cortex 14, 364-375. 
Cabeza, R., Grady, C. L., Nyberg, L., McIntosh, A. R., Tulving, E., Kapur, S., Jennings, J. M., Houle, S., and Craik, F. I. (1997). Age-related differences in neural activity during memory encoding and retrieval: a positron emission tomography study. J. Neurosci. 17, 391-400.

Cohen, M. S. (1997). Parametric analysis of fMRI data using linear systems methods. Neuroimage 6, 93-103.

Cohen-Zion, M., Stepnowsky, C., Johnson, S., Marler, M., Dimsdale, J. E., and Ancoli-Israel, S. (2004). Cognitive changes and sleep disordered breathing in elderly: differences in race. J. Psychosom. Res. 56, 549-553.

Cohen-Zion, M., Stepnowsky, C., Marler Shochat, T., Kripke, D. F., and Ancoli-Israel, S. (2001). Changes in cognitive function associated with sleep disordered breathing in older people. J. Am. Geriatr. Soc. 49, 1622-1627.

Cox, R. W. (1996). AFNI: software for analysis and visualization of functional magnetic resonance neuroimages. Comput. Biomed. Res. 29, 162-173.

Delis, D. C., Kramer, J. H., Kaplan, E., and Ober, B. A. (2000). California Verbal Learning Test, 2nd Edn. San Antonio, TX: Psychological Corporation.

Drummond, S. P. A., and Brown, G. G. (2001). The effects of total sleep deprivation on cerebral responses to cognitive performance. Neuropsychopharmacology 25, S68-S73.

Drummond, S. P. A., Brown, G. G., Gillin, J. C., Stricker, J. L., Wong, E. C., and Buxton, R. B. (2000). Altered brain response to verbal learning following sleep deprivation. Nature 403, 655-657.

Drummond, S. P. A., Meloy, M. J., Yanagi, M. A., Orff, H. J., and Brown, G. G. (2005). Compensatory recruitment after sleep deprivation and the relationship with performance. Psychiatry Res. 140, 211-223.

Eyler Zorrilla, L. T., Jeste, D. V., Paulus, M., and Brown, G. G. (2003). Functional abnormalities of medial temporal cortex during novel picture learning among patients with chronic schizophrenia. Schizophr. Res. 59, 187-198.

Fletcher, P. C., Palomero-Gallagher, N., Zafiris, O., Fink, G. R., Tyler, L. K., and Zilles, K. (2002). The influence of explicit instructions and stimulus material on lateral frontal responses to an encoding task. Neuroimage 17, 780-791.

Forman, S. D., Cohen, J. D., Fitzgerald, M., Eddy, W. F., Mintun, M. A., and Noll, D. C. (1995). Improved assessment of significant activation in functional magnetic resonance imaging (fMRI): use of a clustersize threshold. Magn. Reson. Med.33, 636-647.

Han, S. D., Bangen, K. J., and Bondi, M. W. (2009). Functional MRI of compensatory neural recruitment in aging and risk for Alzheimer's disease: review, and recommendations. Dement. Geriatr. Cogn. Disord. 27, $1-10$.

Howell, A. J., Jahrig, J. C., and Powell, R. A. (2004). Sleep quality, sleep propensity and academic performance. Percept. Mot. Skills 99, 525-535.

Johns, M. W. (1991). A new method for measuring daytime sleepiness: the Epworth sleepiness scale. Sleep 14, 540-545.

Kahn, I., Andrews-Hanna, J. R., Vincent, J. L., Snyder, A. Z., and Buckner, R. L. (2008). Distinct cortical anatomy linked to subregions of the medial temporal lobe revealed by intrinsic functional connectivity. J. Neurophysiol. 100, 129-139.

Kemps, E., and Newson, R. (2006). Comparison of adult age differences in verbal and visuo-spatial memory: the importance of "pure," parallel and validated measures. J. Clin. Exp. Neuropsychol. 28, 341-356.

Lichter, D., and Cummings, J. (2001). Frontal-Subcortical Circuits in Psychiatric and Neurological Disorders. New York, NY: Guildford Press.

Mattis, S. (1988). Dementia Rating Scale: Professional Manual. Odessa, FL: Psychological Assessment Resources Inc.

Mestres-Misse, A., Camara, E., Rodriguez-Fornells, A., Rotte, M., and Munte, T. F. (2008). Functional neuroanatomy of meaning acquisition from context. J. Cogn. Neurosci. 20, 2153-2166.

Middleton, F. A., and Strick, P. L. (2002). Basal-ganglia "projections" to the prefrontal cortex of the primate. Cereb. Cortex 12, 926-935.

Mitchell, K. J., Raye, C. L., McGuire, J. T., Frankel, H., Greene, E.
J., and Johnson, M. K. (2008). Neuroimaging evidence for agendadependent monitoring of different features during short-term source memory tests. J. Exp. Psychol. Learn Mem. Cogn. 34, 780-790.

Nielsen, T., and Levin, R. (2007). Nightmares: a new neurocognitive model. Sleep Med. Rev. 11, 295-310.

Ohayon, M. M., Carskadon, M. A. Guilleminault, C., and Vitiello, M. V. (2004). Meta-analysis of quantitative sleep parameters from childhood to old age in healthy individuals: developing normative sleep values across the human lifespan. Sleep 27, 1255-1273.

Pilcher, J. J., and Walters, A. S. (1997) How sleep deprivation affects psychological variables related to college students' cognitive performance. J. Am. Coll. Health 46, 121-126.

Prince, S. E., Daselaar, S. M., and Cabeza, R. (2005). Neural correlates of relational memory: successful encoding and retrieval of semantic and perceptual associations. J. Neurosci. 25 , 1203-1210.

Qin, S., Rijpkema, M., Tendolkar, I., Piekema, C., Hermans, E. J., Binder M., Petersson, K. M., Luo, J., and Fernandez, G. (2009). Dissecting medial temporal lobe contributions to item and associative memory formation. Neuroimage 46, 874-881.

Reuter-Lorenz, P. (2002). New visions of the aging mind and brain. Trends Cogn. Sci. (Regul. Ed.) 6, 394.

Reuter-Lorenz, P., Stanczak, L., and Miller, A. C. (1999). Neural recruitment and cognitive aging: two hemispheres are better than one, especially as you age. Psychol. Sci. 10, 494-500.

Rosazza, C., Minati, L., Ghielmetti, F., Maccagnano, E., Erbetta, A., Villani, F., Epifani, F., Spreafico, R., and Bruzzone, M. G. (2009). Engagement of the medial temporal lobe in verbal and nonverbal memory: assessment with functional MR imaging in healthy subjects. AJNR Am. J. Neuroradiol. 30, 1134-1141.

Salthouse, T. A., and Ferrer-Caja, E. (2003). What needs to be explained to account for age-related effects on multiple cognitive variables? Psychol. Aging 18, 91-110.

Stenuit, P., and Kerkhofs, M. (2008). Effects of sleep restriction on cognition in women. Biol. Psychol. 77, 81-88.
Stricker, J. L., Brown, G. G., Wetherell, L. A., and Drummond, S. P. A. (2006). The impact of sleep deprivation and task difficulty on networks of FMRI brain response. J. Int. Neuropsychol. Soc. 12, 591-597.

Stringaris, A. K., Medford, N., Giora, R., Giampietro, V. C., Brammer, M. J., and David, A. S. (2006). How metaphors influence semantic relatedness judgments: the role of the right frontal cortex. Neuroimage 33 , 784-793.

Talairach, J., and Tournoux, P. (1988). Co-Planar Stereotaxic Atlas of the Human Brain. New York, NY: Thieme Medical.

van der Veen, F. M., Nijhuis, F. A., Tisserand, D. J., Backes, W. H., and Jolles, J. (2006). Effects of aging on recognition of intentionally and incidentally stored words: an fMRI study. Neuropsychologia 44 2477-2486.

Wechsler, D. (1997). Wechsler Adult Intelligence Scale-III. New York, NY: Psychological Corporation.

Yaffe, K., Blackwell, T., Barnes, D. E., Ancoli-Israel, S., and Stone, K. L. (2007). Preclinical cognitive decline and subsequent sleep disturbance in older women. Neurology 69, 237-242.

Conflict of Interest Statement: The authors declare that the research was conducted in the absence of any commercial or financial relationships that could be construed as a potential conflict of interest.

Received: 29 November 2011; paper pending published: 15 December 2011; accepted: 16 March 2012; published online: 03 April 2012.

Citation: Jonelis MB, Drummond SPA, Salamat JS, McKenna BS, Ancoli-Israel $S$ and Bondi MW (2012) Age-related influences of prior sleep on brain activation during verbal encoding. Front. Neur. 3:49. doi: 10.3389/fneur.2012.00049

This article was submitted to Frontiers in Sleep and Chronobiology, a specialty of Frontiers in Neurology.

Copyright () 2012 Jonelis, Drummond, Salamat, McKenna, Ancoli-Israel and Bondi. This is an open-access article distributed under the terms of the Creative Commons Attribution Non Commercial License, which permits noncommercial use, distribution, and reproduction in other forums, provided the original authors and source are credited. 